\title{
The FAC Experimental Research on Vapor-water Two-phase Flow Piping System of CAP1400 Secondary Loop
}

\author{
Shenhui Zhai ${ }^{1}$, Simin Pang ${ }^{1,}$, Jie Shao ${ }^{1}$, Hongqing Zhu ${ }^{1}$, Xian Zhang ${ }^{1}$ and Pengfei Liu ${ }^{2}$ \\ ${ }^{1}$ State Nuclear Electric Power Planning Design \& Research Institute Co., Ltd, Beijing 100095, China \\ ${ }^{2}$ School of Nuclear Science and Engineering, Shanghai Jiao Tong University, Shanghai 200240, China
}

\begin{abstract}
CAP1400 power plant is one of the advanced Third-generation Pressurized Water Reactor power plant. To ensure the safety and stability of the power plant secondary loop during operation, the corrosion characteristic of CAP1400 secondary loop should been investigated. This paper set up a test facility to study the vapor-water twophase flow corrosion status, which could be seen in the piping system after the control valve of heater drain line. The key parameters, such as pressure drop, temperature, and surface topography have been observed and measured, and the different corrosion characteristic in straight pipe, elbow, tee, and reducer also have been acquired in the tests. The test results and analysis could provide the basis for the corrosion resistance design of the drain pipelines in the CAP1400 nuclear power plant.
\end{abstract}

\section{Introduction}

Nuclear power security is the top priority for the development of nuclear power. One of the most important factors in the design of conventional islands (CI) is flow accelerated corrosion (FAC) which can degrade the pipeline material even cause pipeline rupture [1-2]. Since late 1970s, several serious pipeline rupture accidents have occurred in the $\mathrm{CI}$ of some pressurized water reactor (PWR) nuclear power stations in the United States. These incidents have attracted the attention of the United States Nuclear Regulatory Commission and the nuclear industry. A special investigation team was established to investigate these incidents. The results of the investigation showed that that these pipeline failures were caused by FAC. Subsequently, many countries around the world also began conducting extensive research on the FAC. Unit 2 of the Arkansas Nuclear One Nuclear Power Plant in the US is a PWR unit owned by Combustion Engineering. In 1989, a rupture occurred at the welding place between the high-pressure steam extraction interface of the turbine and the extraction pipeline, as shown in Figure 1. The accident was the FAC damage that took place in the two-phase flow system since the Surry power station accident, which also reminded the nuclear power practitioners to strengthen their understanding of the destruction of two-phase flow FAC.

Chinese CAP1400 demonstration project will build two $1500 \mathrm{MWe}$ class PWR units; the design life is 60 years. CI adopts half-speed condensate TurbineGenerator unit. There are seven stages of regenerative feedwater heaters in secondary loop, which is consist of four stages LP heaters, two stages HP heaters and one deaerator. [3]

In the secondary loop of the CAP1400 nuclear power plant, a large number of vapor-water two-phase flow phenomenons exist. The pipe downstream of the heater drain control valve is a typical vapor-water two-phase flow pipeline. For the corrosion research and protection of the vapor-water two-phase flow pipelines in the secondary loop, no relevant regulations and rules can be followed in China, and the corrosion research of the vapor-water two-phase flow pipelines under the simulated actual working conditions is still in its infancy. This paper adopted experimental methods to study the FAC of the typical vapor-water two-phase flow pipelines in secondary loop in order to provide the basis for design and corrosion protection of the vapor-water two-phase flow pipelines of the subsequent CAP1400 nuclear power plants.

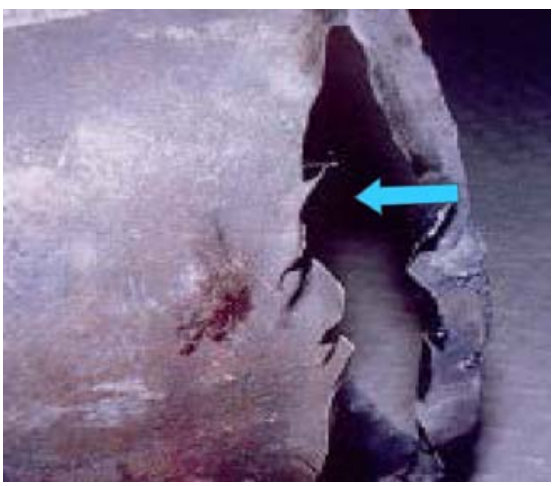

Figure 1. Cracked Welded Joint of Unit 2 of the Arkansas Nuclear One Nuclear Power Plant.

a Corresponding author: pangsimin@snpdri.com 
FAC is a type of global corrosion, which is usually accelerated and deteriorated due to the medium flow. FAC is mainly formed by the interaction of physical and electrochemical processes. It is worth paying attention that FAC works through dissolution process rather than mechanical destruction of the oxide layer which is different from the common erosion/corrosion (EC) mechanism [4]. Moreover, the dissolution takes effect only when the medium is water. Therefore, the precondition of $\mathrm{FAC}$ is that the flowing medium in the pipeline is water or wet steam.

FAC formation is mainly divided into two processes: the first is the dissolution process of metal surface, and the second is the diffusion process of dissolved products.

Due to the oxidation reaction between iron atoms of the steel pipe and the water molecules of the flowing water in the pipe, oxide layers are formed which consist of an inner layer that grows inwards the metal matrix and a relatively loosely attached outer layer. Both layers are mainly composed of $\mathrm{Fe}_{3} \mathrm{O}_{4}$. When there are oxygen molecules in the water, $\mathrm{Fe}_{2} \mathrm{O}_{3}$ can also be formed which can strengthen the structure of the $\mathrm{Fe}_{3} \mathrm{O}_{4}$ oxide layer to make it denser. The formation of oxide layer can avoid direct contact between iron atoms of metal matrix and water molecules, thereby protecting the metal matrix. However, due to the oxide layer produced by natural reaction cannot form a dense structure that completely covers the surface of metal matrix, the pores and other discontinuous structures are inevitable in the formation of the oxide layer. These places become the weak points of the oxide layer, which makes the flowing medium and the metal matrix interacts continuously through the pores, resulting in the dissolution of the metal matrix and the oxide layer. The structure of the oxide layer formed on the metal surface is porous, therefore ferrous ions can diffuse through pores to the medium (water or wet steam). In three areas (main stream area, boundary layer, and oxide layer), the processes of dissolution and diffusion of ferrous ions are continuous. The ferrous ions produced by the corrosion of the metal matrix diffuse to the boundary layer outside the oxide layer through the pores, while the flowing water carries away these ferrous ions which are just diffused to the main stream area.

The FAC can cause a more obvious corrosion pattern on the inner surface of the metal pipeline. Due to the different state of the flowing medium in the pipeline, the corrosion forms are also different. In general, FAC can be divided into two types, single-phase flow FAC and twophase flow FAC.

Under single-phase flow condition, FAC will cause horseshoe shaped pits on the metal surface, and the entire surface looks like an orange peel, as shown in Figure 2. In some areas where FAC rate is not very high, there are obvious dent features. If further investigation is conducted, it will be clear that these features have a certain direction and can be described as "serrated" or "horseshoe-shaped" pit "strings" whose tips point in the direction of flow [5]. As the corrosion becomes more severe, these serrated shapes gradually overlap. The most serious corrosion parts will create continuous rounded holes or orange-peel-shaped abrasions. Almost no oxide $\left(\mathrm{Fe}_{3} \mathrm{O}_{4}\right)$ remains on the surfaces of these corrosion parts, and it can be seen on the metal profile that the oxide layer is very thin (about a few microns). Using a microscope scan with a higher magnification ratio, the structure of a typical rounded dent caused by FAC can be observed.

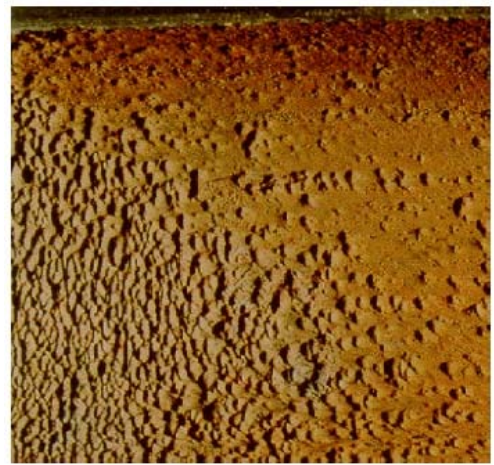

Figure 2. Corroded Metal Surface Caused by Single-phase FAC.

Under two-phase flow condition, FAC will cause tiger print shaped appearance on the metal surface, as shown in Figure 3. The black part of the figure is the area with relatively serious corrosion while the red part of the figure is the oxide layer on the metal surface. The twophase FAC usually creates the wear marks on the metal surface which appear black and shiny, or even enamellike. These marks usually have pit-like markings, which sometimes show serrated or horseshoe-like directional characteristics as the damage caused by single-phase flow.

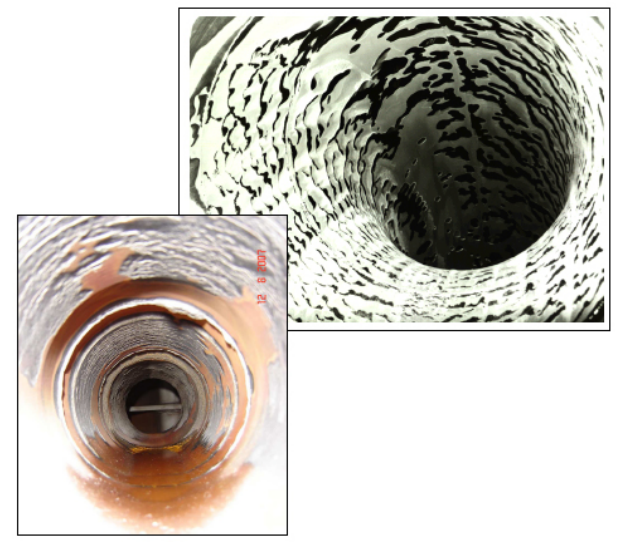

Figure 3. Corroded Metal Surface Caused by Two-phase FAC.

\section{Experimental Methods}

The research results on the influencing factors of FAC show that FAC has many influencing factors, such as temperature, $\mathrm{pH}$ value, velocity, and rate of vapor content of the medium etc. If the design condition of the secondary loop is fixed, some influencing factors are determined, such as $\mathrm{pH}$ valve, velocity, temperature, etc., which also brings convenience for experimental research of two-phase FAC. In this paper, the pipeline downstream of a heater drain control valve in the secondary loop of the CAP1400 nuclear power plant was taken as the test object. A two-phase flow erosion-corrosion facility (TPEC) was designed and built for experimental research, and the design parameters of the pipeline are shown in Table 1 . 
Table 1. Experimental Parameters of Vapor-water Two-Phase Flow in CAP1400 CI Pipeline.

\begin{tabular}{|c|c|c|c|c|}
\hline Name & $\begin{array}{l}\text { Operating } \\
\text { Pressure }\end{array}$ & $\begin{array}{l}\text { Operati } \\
\text { ng } \\
\text { Temper } \\
\text { ature }\end{array}$ & $\begin{array}{c}\text { Veloci } \\
\text { ty }\end{array}$ & $\begin{array}{c}\text { Piping } \\
\text { Material } \\
\text { After the } \\
\text { Drain } \\
\text { Valve }\end{array}$ \\
\hline $\begin{array}{l}\text { Normal } \\
\text { Drain } \\
\text { Pipe of a } \\
\text { High- } \\
\text { Pressure } \\
\text { Heater }\end{array}$ & $\begin{array}{c}\text { Upstream of } \\
\text { the Drain } \\
\text { Control } \\
\text { Valve: } \\
1.867 \mathrm{MPa} \\
\text { Downstream } \\
\text { of the Drain } \\
\text { Control } \\
\text { Valve: } \\
1.026 \mathrm{MPa} \\
\end{array}$ & $184.5^{\circ} \mathrm{C}$ & $\begin{array}{l}1.29 \\
\mathrm{~m} / \mathrm{s}\end{array}$ & TP304L \\
\hline
\end{tabular}

The TPEC experimental device is a closed loop, which consists of a high-pressure (HP) tank (design pressure is $4 \mathrm{MPa}$, and design volume is $2.5 \mathrm{~m} 3$ ), a lowpressure (LP) tank (design pressure is $0.4 \mathrm{MPa}$, and design volume is $10 \mathrm{~m} 3$ ), a pump, a heat exchanger, intube heaters, test section, measurement and control system, and auxiliary system. The pressure control of the test section is jointly accomplished by the pump, the pressure control system of the HP tank, and the test section control valve: the pump with variable voltage and variable frequency (VVVF) system allows for coarse adjustment of pressure and flowrate; the pressure control system on the top of the HP tank can adjust and stabilize the pressure of the loop; the control valve before the test section is used for precise adjustment of the pressure before the test section; the downstream pressure of the pipeline is adjusted by the pressure control system of the LP tank. The temperature control of the loop is jointly accomplished by the in-tube heater, the in-tank heater of HP tank, and the preheater before the test section: the intube heater with a rated power of $600 \mathrm{~kW}$ which can be adjusted is used for adjusting the inlet temperature of the HP tank; the in-tank heater with a rated power of $200 \mathrm{~kW}$ is used for adjusting the outlet temperature of the HP tank; the preheater with a rated power of $50 \mathrm{~kW}$ is used for precise adjustment of the inlet temperature of the test section to ensure the inlet temperature condition of the test section. A bypass pipeline of the test section is installed to regulate the flowrate and ensure the flow velocity in the test section. After the high-temperature and high-pressure water flashes to vapor-water mixture in the test section, it is pumped to the heat exchanger with a rated power of $430 \mathrm{~kW}$ to be cooled down, then it flows back to the LP tank.

The specification of the pipe in the test is DN50. The material after the control valve is determined according to the test conditions, and the material of other parts of the test loop is 304 stainless steel.

The schematic diagram of TPEC test loop is shown in Figure 4.

Commonly used pipe fittings of the secondary loop include straight pipes, elbows, tees, and reducers, etc. Therefore, these typical pipe fittings were measured for the experiments. Considering the experimental conditions, the design parameters of the test section are shown in Table 2. The test period is designed to be 2 hours.

Table 2. Design Parameters of the Test Section.

\begin{tabular}{|c|c|c|}
\hline Type & Specification & Material \\
\hline $\begin{array}{l}\text { Straight Pipe } \\
\text { Test Section }\end{array}$ & $\begin{array}{c}\text { Diameter: DN50; } \\
\text { Wall thickness: } 7 \mathrm{~mm} \text {; } \\
\text { Length: } 2.5 \mathrm{~m}\end{array}$ & $\begin{array}{l}\text { No. } 20 \text { Steel/ } \\
\text { TP304L }\end{array}$ \\
\hline $\begin{array}{l}90^{\circ} \text { Elbow } \\
\text { Test Section }\end{array}$ & $\begin{array}{l}\text { Pipe Diameter: DN50; } \\
\text { Bend Radius: } 200 \mathrm{~mm}\end{array}$ & $\begin{array}{c}\text { No. } 20 \text { Steel / } \\
\text { TP304L }\end{array}$ \\
\hline $\begin{array}{l}\text { Reducer Test } \\
\text { Section }\end{array}$ & $\begin{array}{l}\text { Pipe Diameter before } \\
\text { Control Valve: DN50; } \\
\text { Pipe Diameter after } \\
\text { Control Valve: DN80; }\end{array}$ & $\begin{array}{l}\text { No. } 20 \text { Steel / } \\
\text { TP304L }\end{array}$ \\
\hline $\begin{array}{l}\text { Tee Test } \\
\text { Section }\end{array}$ & $\begin{array}{c}\text { Pipe Diameter: } \\
\text { DN50×DN50×DN50 }\end{array}$ & $\begin{array}{c}\text { No. } 20 \text { Steel / } \\
\text { TP304L }\end{array}$ \\
\hline
\end{tabular}

\subsection{Straight Pipe Test Section}

In the test, a pipe with a nominal diameter of $50 \mathrm{~mm}$ is adopted to perform surface analysis on the inner surface of the pipe. Measuring points and surface analysis test sections are set up at equal distance on the test pipe, which can observe and analyze the scour morphology on the pipe wall.

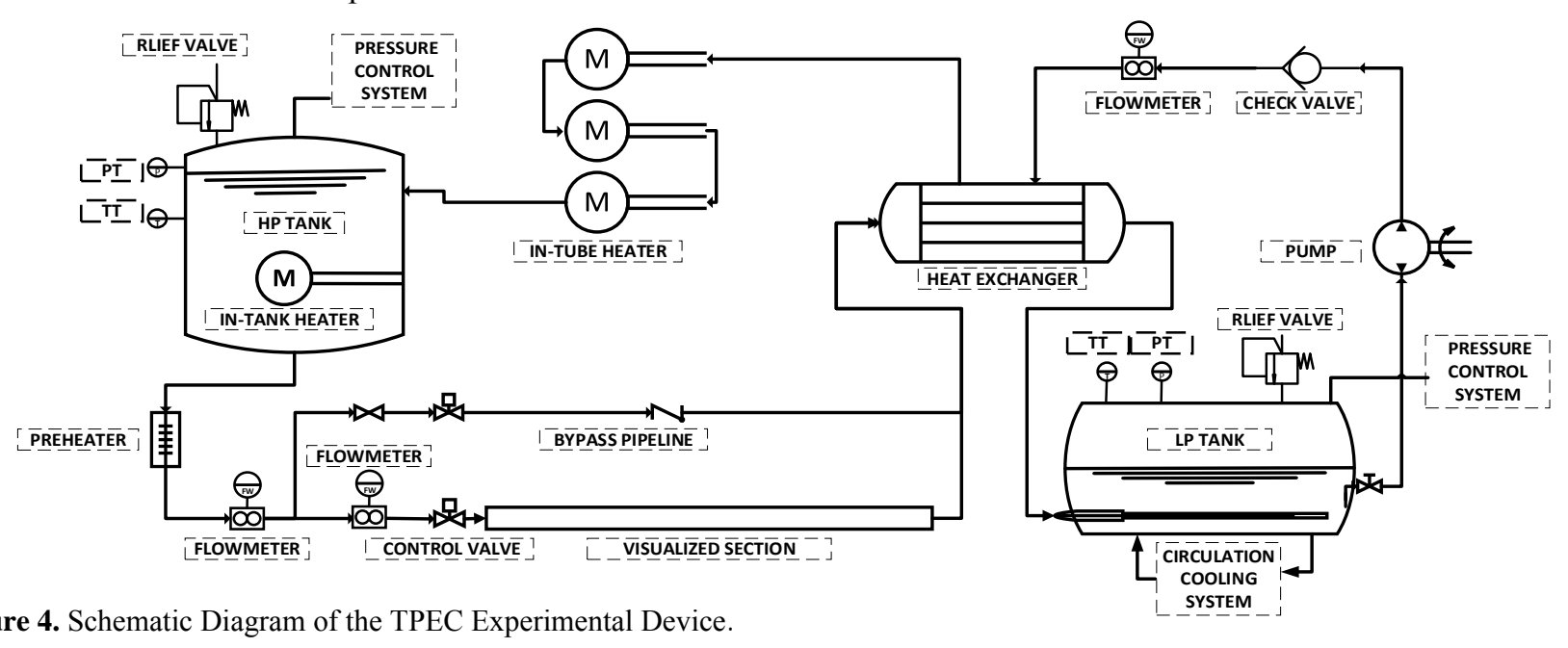

Figure 4. Schematic Diagram of the TPEC Experimental Device. 


\subsection{Elbow Test Section}

The bend radius of the test section is $200 \mathrm{~mm}$, and the diameter of the pipe is $50 \mathrm{~mm}$. The test locations are designed at $5^{\circ}, 25^{\circ}, 45^{\circ}, 65^{\circ}$, and $85^{\circ}$ of the elbow. The test piece is fixed at the top of each test location. The test piece is replaceable, which is fixed to the measuring point by using the tighten bolt. The outer sleeve and the tighten bolts are threaded connected. There is a pressure measuring point and a temperature measuring point on the test section.

\subsection{Reducer Test Section}

Since the reducers are necessary components for connecting the pipes with different diameters. The existence of pipe expansion in the secondary loop of the nuclear power plant can also be one of the concerns of the two-phase flow scour test. In the test, the DN50 $\times 80$ reducer is adopted, and the length of the reducer is $100 \mathrm{~mm}$. The scouring effect of the two-phase flow on the wall of the expansion part is observed. The reducer test section is designed to be replaceable, which is similar to the straight pipe test section. The test pieces are positioned through the positioning component and fixed by the threaded tighten pipe. The test pieces can be replaced by unscrewing the threaded tighten pipe in order to save the processing time of the test pieces.

\subsection{Tee Test Section}

Tee is one of the common components used in the piping system after which the flow pattern and velocity of the fluid change greatly. Therefore, the vapor-water two phase flow can also result in corresponding wear that different from other piping components on the tee. In the test, a standard DN50 equal tee is adopted, and the design of dismantling and replacement are also considered. The replaceable test pieces used in this test are with the same design as the pieces used in straight pipe test section.

\section{Experimental Results and Analysis}

Based on the parameters of the pipe after the heater drain control valve in the secondary loop of a CAP1400 nuclear power plant which is under construction, the twophase flow corrosion experiments were conducted for typical piping components including straight pipe, elbow, tee, and reducer. The main experimental results are as follows.

\subsection{Measurement of Thermodynamic Parameters of Two-Phase Flow}
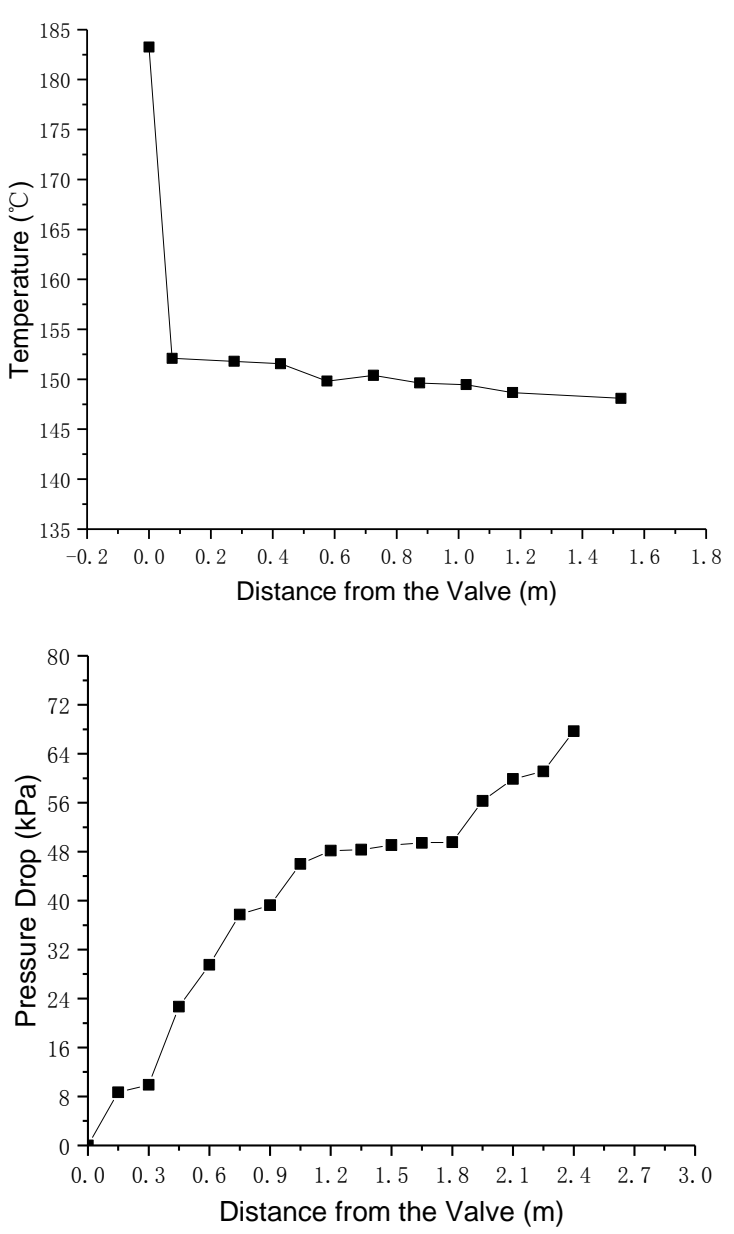

Figure 5. Curve of Temperature and Pressure Drop along Straight Pipe Section.

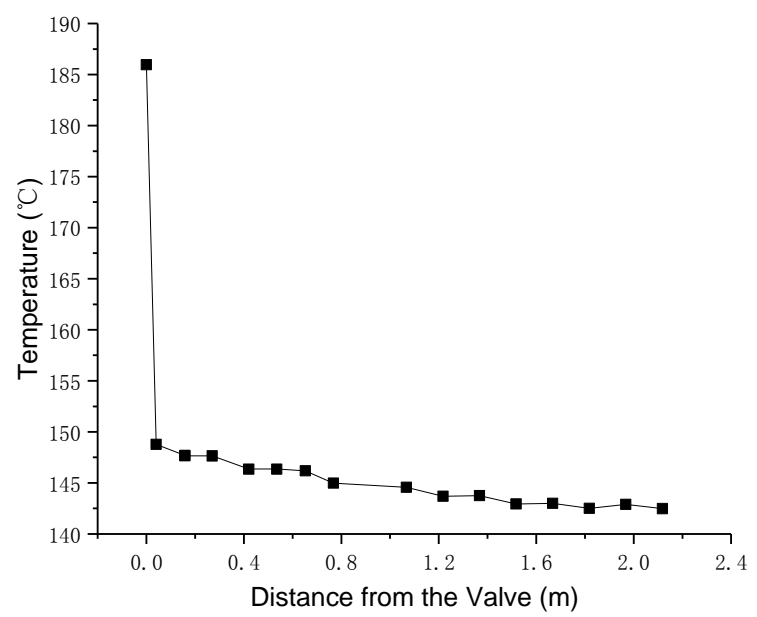




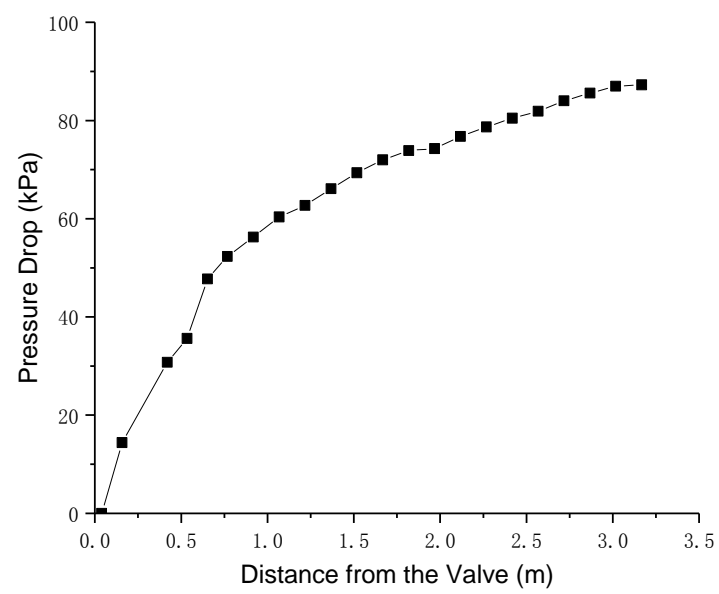

Figure 6. Curve of Temperature and Pressure Drop along Elbow Section.
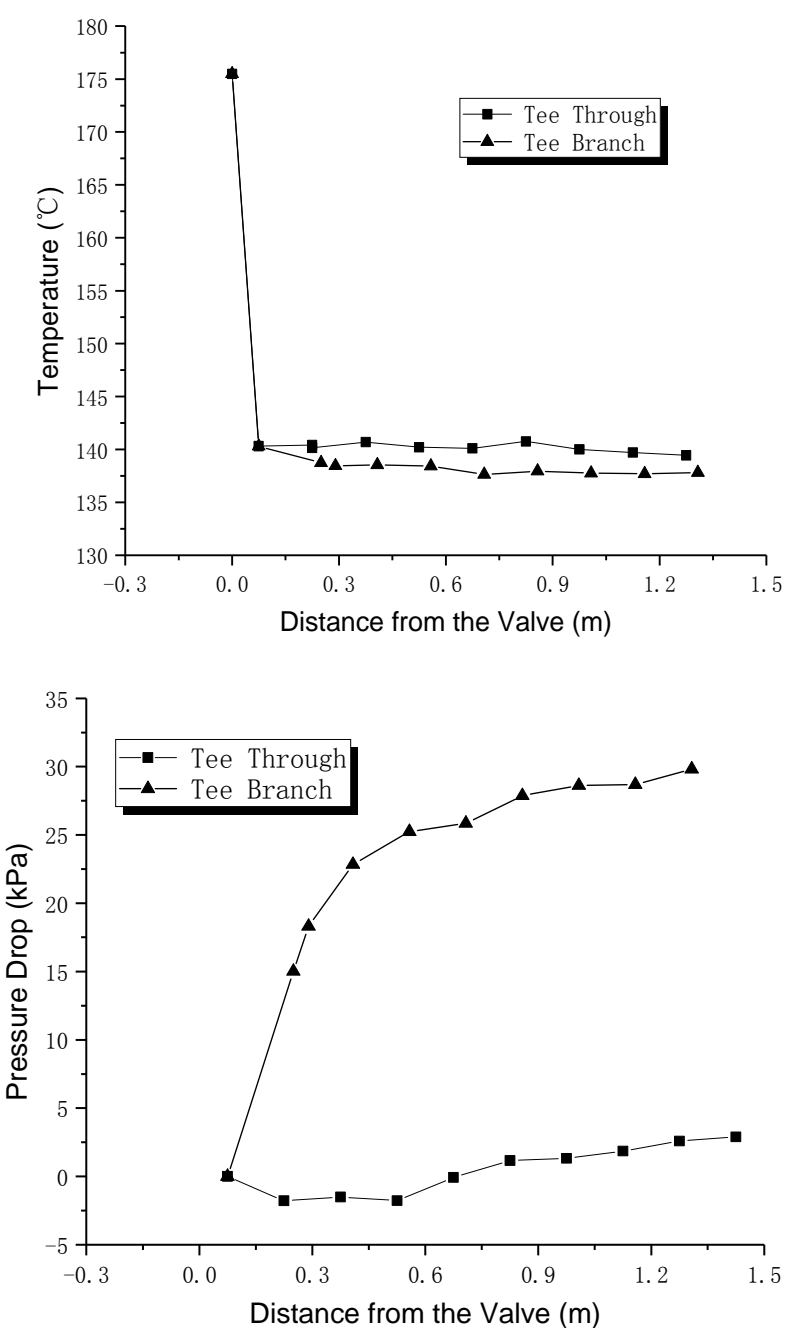

Figure 7. Curve of Temperature and Pressure Drop along Tee Section.
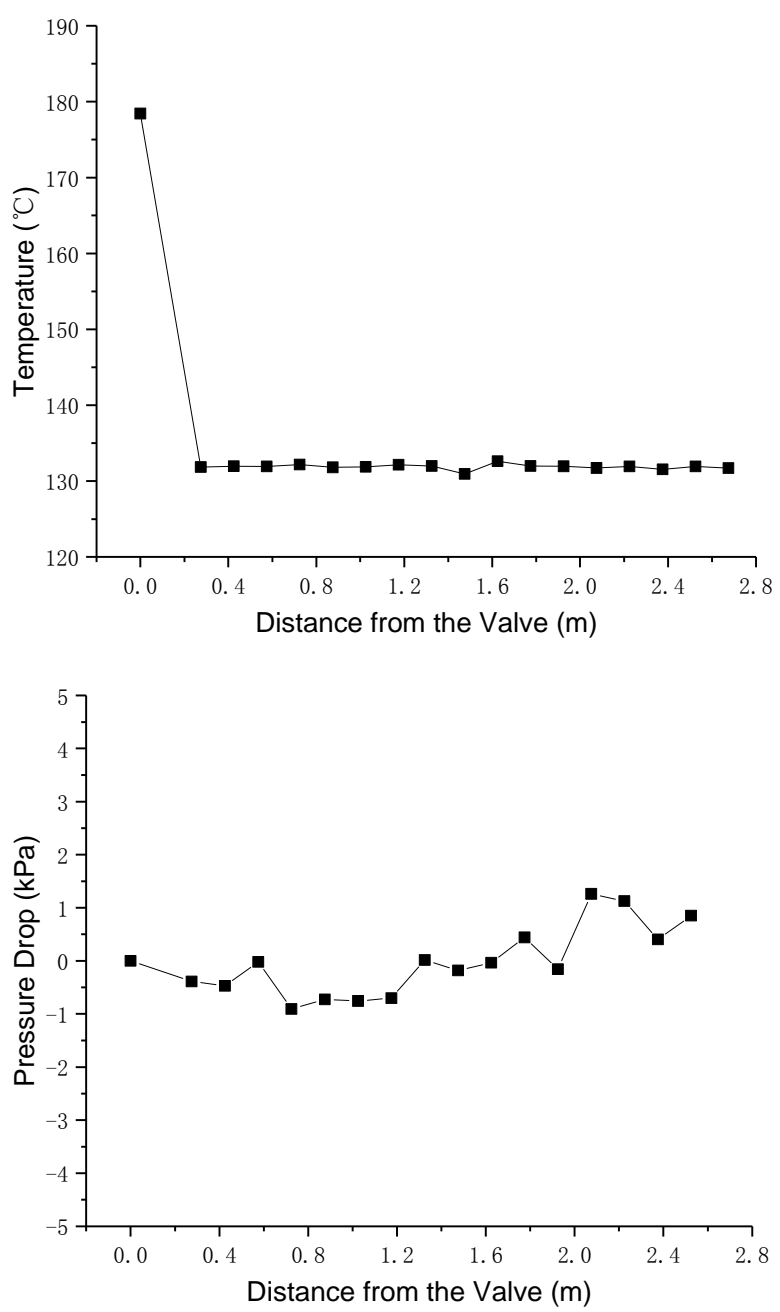

Figure 8. Curve of Temperature and Pressure Drop along Reducer Section.

The curves of temperature and pressure drop along straight pipe section, elbow section, tee section and reducer section under the condition listed in Table 1 were measured, as shown in Figure 5 8. As can be seen from the figure, after passing through the valve, due to the pressure drop, there will be a stepped steep drop in the temperature after the valve which is related to the form of the piping components. The temperature of the straight pipe section after the control valve is the highest while the temperature of the reducer section after the control valve is the lowest. The reason is that the straight pipe has the least influence on the flow pattern. However, for the reducer section, before entering to the pipe with larger diameter, the flow of the fluid is driven by the pressure difference, which means the fluid moves under favorable pressure gradient. When the fluid enters the area with a larger diameter, based on the mass continuity, the velocity of the fluid will decrease due to the increase of the flow area. According to the Bernoulli equation of incompressible fluid, the decrease of velocity will lead to the increase of static pressure, which will result in appearance of adverse pressure gradient in the local area on upper and lower sides of the reducer. The combined effect of adverse pressure gradient and wall viscous resistance will cause the fluid in the local area to decelerate continuously. Eventually, the inertial force 
cannot overcome the resistance and cause the flow to leave from the wall surface and generate vortex. Since the velocity of the viscous fluid does not change abruptly as the pipe shape, it changes gradually. This sudden change in the flow distribution results in severe turbulence in the local area, which will reduce the pressure in the local area and cause the temperature to drop rapidly. The temperature change of elbow and tee is between the straight pipe and reducer.

For the pressure drop along the distance, the change trend of straight pipe, elbow, and tee is basically the same. The pressure drop increases along with the distance, and it tends to be constant when the distance is long enough. According to the experimental results, the pressure drop of the elbow is larger than that of the straight pipe. Because of the diversion effect of the branch pipe, the flow of the tee cannot be compared with the straight pipe or the elbow directly. Therefore, it can be qualitatively considered that the pressure drop with flow pattern change is larger than that of the straight pipe. It can be seen from the figure that the pressure drop of the reducer along the distance tends to 0 . It can be considered that the development area of the two-phase flow is extremely short and the interaction between two phases is small, therefore the flow pattern reaches the relatively stable state which is different from the straight pipe and elbow that need certain space for development to achieve a stable state.

According to the above experimental results, expanding the pipe diameter of the downstream pipe of the drain control valve by reducer is beneficial for the thermodynamic parameters of the fluid in the pipe after the control valve.

\subsection{Morphology of the Surface Corroded by Two- Phase Flow}

According to the sample size and surface scour marks, the scanning electron microscope (SEM) and energy dispersive spectrometer (EDS) were employed to scan the sample surface and the surface morphology of the samples were measured by a three-dimensional profilometer. In order to better observe the corrosion patterns in the experiments, samples made of different materials were used, one is made of No. 20 steel (carbon steel) and the other is made of TP304L (stainless steel). Since the corrosion effect of TP304L test samples is not obvious, the test results of carbon steel samples are listed only.

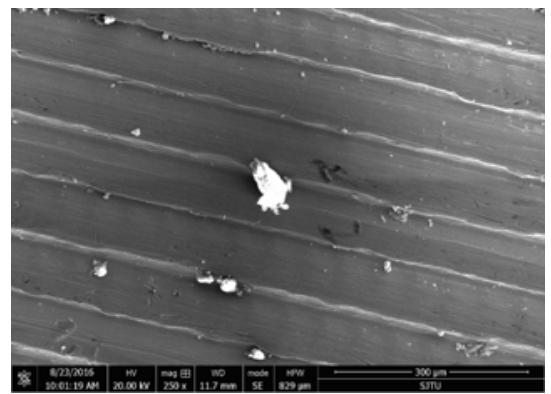

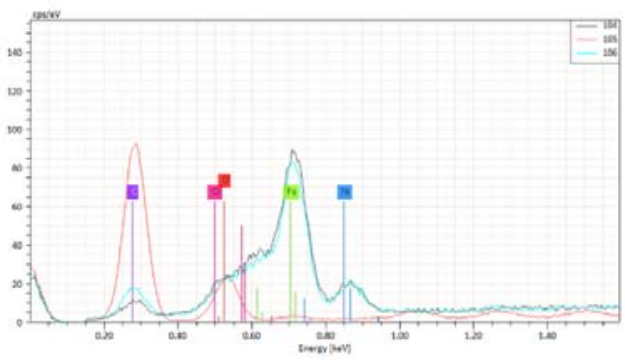

Figure 9. Surface Morphology and Element Distribution of Carbon Steel Sample in Straight Pipe Test Section.
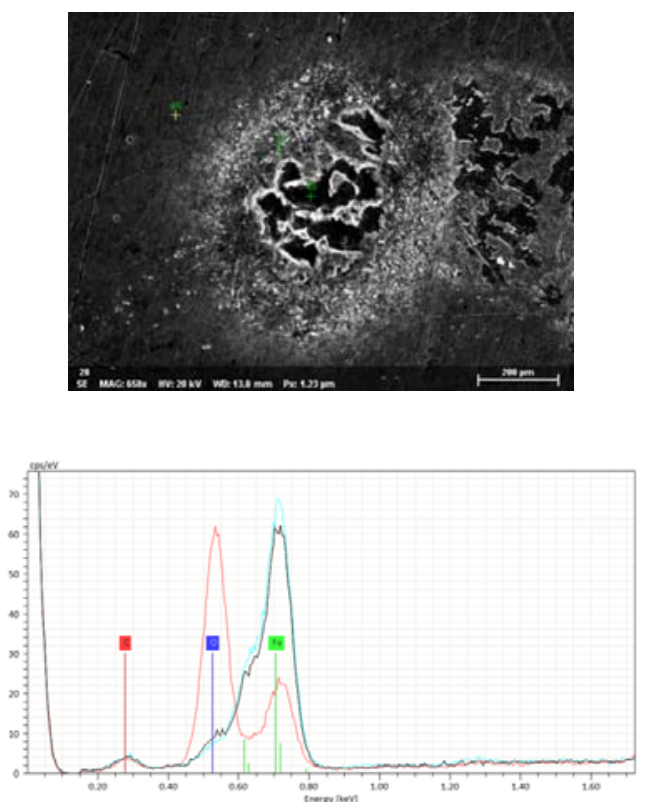

Figure 10. Surface Morphology and Element Distribution of Carbon Steel Sample in Elbow Test Section.
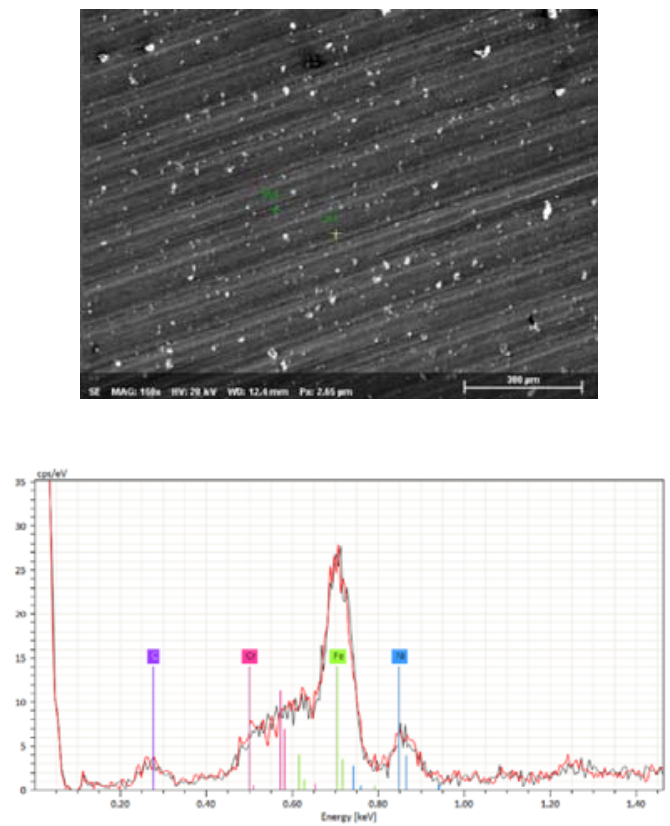

Figure 11. Surface Morphology and Element Distribution of Carbon Steel Sample in Tee Test Section. 

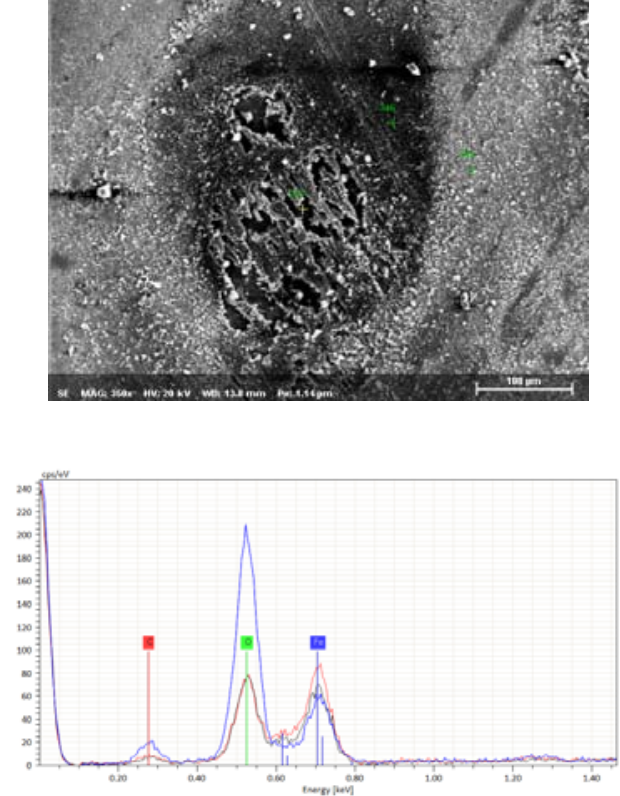

Figure 12. Surface Morphology and Element Distribution of Carbon Steel Sample in Reducer Test Section.

Figure. $9 \sim$ Figure. 12 show the surface morphology and elemental analysis results of carbon steel samples tested in each experiment of straight pipe, elbow, tee, and reducer. As can be seen from Figure 9, after the carbon steel samples scoured by two-phase flow in straight pipe section, the oxide concentration area can be observed. The concentration area is basically round, the size of it is ranging from $0.5 \sim 1 \mathrm{~mm}$. According to the EDS observation, no oxygen can be found out of the oxide concentration area. In the oxide concentration area, the proportion of oxygen is different in different area, the thickness of oxide layer is thicker in the center of the oxide concentration area than at the edge. The experimental results show that there is a significant concentration of oxygen element and a significant increase of carbon element in the destroyed area of the sample which means high temperature oxidation occurs at the place that result in embrittlement of the structure.

In Figure 10, it can be seen more clearly in the sample of elbow test that obvious round shape scour marks are showed on the surface of the No.20 steel sample, and a few dotted pits are generated inside these round shape scour marks. These pits are irregular in position, but mainly concentrated inside of the scour marks. The larger marks have more pits inside of them and the pit size is smaller in the smaller scour marks.

From Figure 11, it can be seen that the samples of tee test section can also be scoured, and there are round scour marks appear on the surface which looks less obvious than the scour marks on the samples of the straight pipe test and the reducer test. Considering the position of the samples, it can be considered that the two-phase flow area in the tee is relatively large, and thermodynamic parameters change significantly at the position of the tee (which due to the flow diversion caused by the branch pipe). Therefore, the two-phase effect is weakened, causing less corrosion.
As shown is Figure 12, the scour marks of the size between $0.1 \sim 0.3 \mathrm{~mm}$ are also found on the surface of the samples of reducer section. The structural failure points are showed in the internal area of the scour marks. The failure points appear to be typical FAC morphology, and the material is scales off the surface. According to the EDS results, oxygen element is found in the damage point which indicates that oxidation also occurs at the damage point. And the oxide layer inside the scour marks appears the trend of thinning. It means that the dotted pits produced by the two-phase flow are generally dotted pits, which usually appears inside the round shape scour marks. The scour marks are created by the high temperature generated by the bubbles impact, which leads to the property change or thinning of the oxide layer. And the oxide layer is delaminated due to the bubble scouring or the mechanical force produced by the bubble collapse.

Under the same experimental conditions, no obvious scour marks are seen on the surface of the TP304L steel samples.

\subsection{The Correlation between the Thinning of Piping Wall thickness and the Scouring Time}

In order to observe the correlation between the thinning of the piping wall thickness and the time, the impact experiments of scouring time were conducted. Two sample points (point 1 is at the $0^{\circ}$ of the elbow, point 2 is at the $30^{\circ}$ of the elbow) were measured using a threedimensional profilometer, and the test results are as follows.

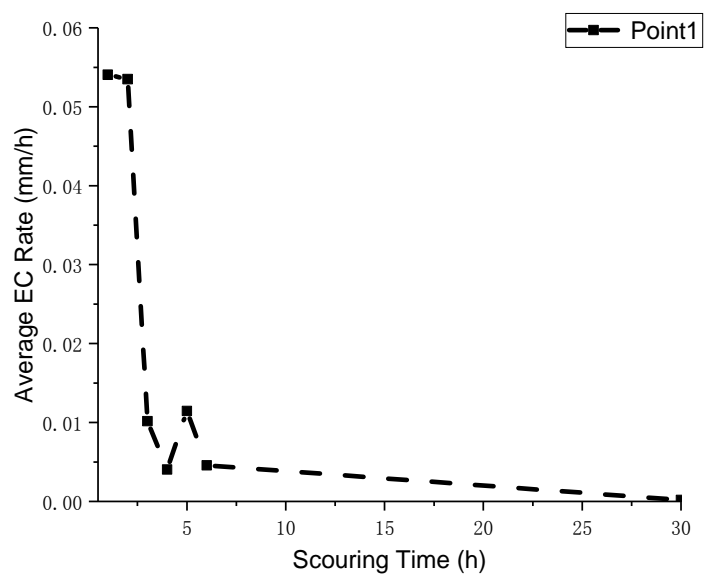

Figure 13. The Curve of the Average Piping Wall Thickness Reduction of the Samples at Point 1 of the Elbow Test Section with Time.

It can be seen from Figure 13 14, the erosion rate was larger during the initial $1 \mathrm{~h} \sim 2 \mathrm{~h}$, which was related to that the initial piping wall was already oxidized. The average EC rates are significantly different for the two sample points is due to the different initial oxidation degree. After the initial oxidation layer was scoured away, the erosion rate dropped rapidly, but it increased at $5 \mathrm{~h}$. This is related to the process of erosion development. During the initial period of two-phase scouring, the pipe wall oxidized and generated the oxide layer in the water 
environment. Due to the low strength of the oxide layer, it was stripped away from the pipe surface by the twophase flow bubbles and left several dotted pits. Since the peeling of the oxide layer exposed the underlying metal matrix which strength is relatively high and hard to be damaged by the two-phase flow bubbles, the erosion rate starts to decrease. With continuous oxidation of the metal matrix, the erosion rate increases slightly at later time.

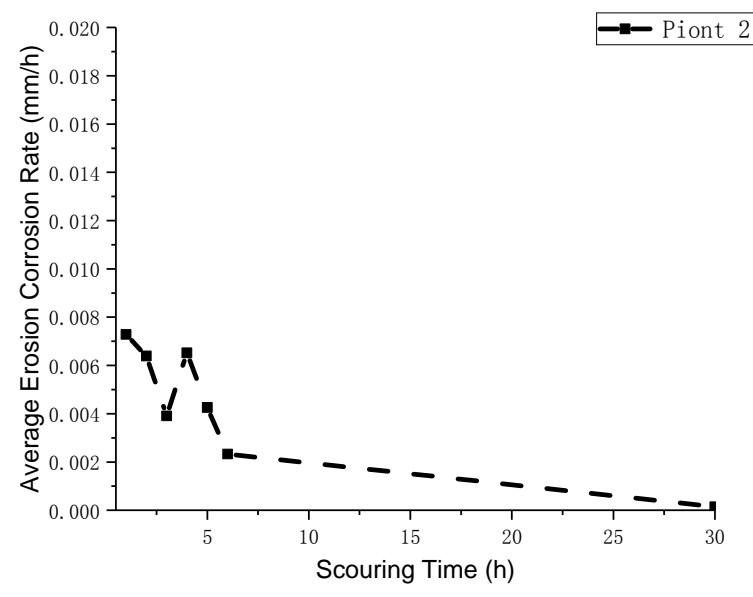

Figure 14. The Curve of the Average Piping Wall Thickness Reduction of the Samples at Point 2 of the Elbow Test Section with Time.

According to the experimental results, due to the reduction of the oxygen concentration of the fluid in the pipeline, the piping wall thickness reduction rate reached a relatively small steady value after a period of time, which was about $0.485 \mu \mathrm{m} / \mathrm{h}$.

\section{Conclusions}

In this paper, the TPEC experimental device was used to study the FAC effects of the pipeline after the drain valve in the CAP1400 secondary loop. The thermodynamic parameters of two-phase flow and the corrosion morphology of the pipe surface in different straight pipe sections, elbow sections, tee sections and reducer sections were measured. The following conclusions can be drawn from the experimental measurement and analysis:

(1) Due to the pressure drop, the high-temperature and high-pressure water will be vapor-water two-phase fluid after flowing through the drain valve. The thermodynamic parameters are related to the parameters before the drain valve. Under the same before-valve thermodynamic parameters, the energy loss of the straight pipe is smaller so that the temperature after valve of it is the highest; the flow development is faster and the interaction between two phases is relatively small in the reducer so that the temperature after valve of it is the lowest.

(2) The corrosion mechanism is the vapor phase of the vapor-water two-phase flow has an impact on the oxide layer which peels off the oxide layer from the pipe surface and exposes the metal matrix to the water to be further oxidized, therefore, the erosion-corrosion continues.

(3) According to the measurement results of the threedimensional profilometer, the rate of the corrosion caused by vapor-water two-phase flow scouring follows a special law: at the beginning of the corrosion, the oxide layer becomes thicker due to the continuously oxidation, however, the impact from bubbles makes the oxide layer easier to be peeled so that the corrosion rate is faster at the initial period. With scouring continuing, the loose oxide layer is washed away and the corrosion rate decreases. However, the dotted pits created by the scouring expose the metal matrix, so that the oxidation proceeds and the corrosion rate increases. It can be seen that the erosion-corrosion and the metal oxidation interact on the pipeline together, therefore, no obvious erosioncorrosion marks were observed on the 304L stainless steel which has a strong resistance to oxidation

(4) At the initial period of the two-phase flow, the fluid can facilitate the formation of the oxide layer. After the oxide layer is formed, the oxide layer separates the metal matrix from the fluid so that the corrosion rate of the pipe wall tends to be stable.

\section{References}

1. Derek H. Lister, L. liu, A. Feicht, M. Khatibi, W. Cook, K. Fujiwara, and E. Kadoi. A Fundamental study of flow-accelerated corrosion in feed water systems, 15th International Conference on the Properties of Water and Steam Water, Steam, and Aqueous Solutions: Advances in Science and Technology for Power Generation, ICPWS XV, Berlin, Sept. 8-11, (2008).

2. Recommendations for Controlling Cavitation, Flashing, Liquid Droplets Impingement, and Solid Particle Erosion in Nuclear Power Plant Piping Systems, EPRI, 1011231, 2004.

3. Chenge Lin, Zusheng Yu. Advanced passive pressurized water reactor technology. Beijing, Atomic Energy Press, 243-246, (2009).

4. Bryan Poulson, Complexities in predicting erosion corrosion, Wear: 497-507, (1999).

5. Barry Dooley, Flow accelerated Corrosion in Fossil and Combined Cycle/HRSG Plants: A summary. Lyon: FAC2008 International conference, 961-975 (2008). 